\title{
Numerical Simulation and Design of Computer Aided Diabetic Retinopathy Using Improved Convolutional Neural Network
}

\author{
Lokesh Gupta $^{1}$ and Dr. Saroj Hiranwal ${ }^{2}$ \\ 1M.Tech. (Scholar) Department of Computer Science and Engineering \\ RIET, Jaipur, Rajasthan, India \\ 2 Professor, Department of Computer Science and Engineering \\ RIET, Jaipur, Rajasthan, India
}

\begin{abstract}
The health sector is entirely different from other sectors. It is a high priority department with the highest quality of care and quality, regardless of cost. It does not meet social standards even though it absorbs a lot of budget. Health specialists interpret much of the medical evidence. Due to its subjectivity, complexity of images, broad differences among various interpreters and exhaustion, the image interpretation of human experts is very restricted. It also offers an exciting solution with good medical imaging accuracy following in-depth learning in other practical applications and is considered an important tool in future healthcare applications. This chapter addresses the most advanced and optimised deep learning architecture for segmentation and classification of medical pictures. We addressed the complexities of healthcare imaging and open science based on profound learning in the previous segment.

Diabetic retinopathy automated diagnosis is crucial because it is the primary cause of permanent vision loss in working-age people in developed countries. The early identification of diabetic retinopathy is extremely helpful in clinical treatment; although many different methods of extracting functions were suggested, the classification task of retinal images is still quite tedious for even those professional clinicians. Recently, in contrast with previous feature-based image-classification approaches, deep-convolutioned neural networks have demonstrated superior performance in image classification. Therefore in this research, we explored the use of deep-seated neural network techniques to identify diabetic retinopathy automatically with Color Fundus images in our datasets that are superior to classical ones.

Deep convolutionary neural systems have since late been seen better output in the analysed image arrangement than previous components which have combined image order techniques that are focused on the crafting method. In this investigation, we studied the use of profound convolutionary strategy of the neural system to naturally classify diabetic retinopathy, using shading fundus images to achieve high precision in our datasets.
\end{abstract}

Keywords - Diabetic Retinopathy, Deep Learning, Machine Learning, CNN, Fundus, Image.

\section{INTRODUCTION}

Diabetes can be considered a persistent rise in blood glucose and one of the world's most rapidly expanding health threats $[9,10]$. There are approximately 150 to 200 million diabetes diagnosed, about 50 million of whom are diagnosed alone in Europe [3]. Moreover many people are still sad. In Finland with a population of approximately 5 million, there are 280,000 diabetes treatments, 40,000 persons (type 1 diabetes) experience permanent injuries from insulin development on isles, and a rise in insulin resistance in 240,000 people (type 2 diabetes) [08]. The latest forecast also predicts that 200000 patients are not diagnosed and that the number of diabetes patients will double every 12 years. These startling facts encourage prevention and screening methods for the large populations, since diabetes is adequately and earlyly in treatment [11].

Digital imaging technology has developed into a multi-purpose, non-invasive measuring tool for a variety of medical applications. Latest practise in many eye clinics is the imaging of the fundus with new technology and it becomes more important with rising life expectancy and healthcare costs. Diabetic retinopathy is the common complication of diabetes, as the retina is susceptible to diabetes microvariety alteration, so the fundus imaging procedure is a non-invasive and painless pathway by which diabetic eyes are screened and monitored.

As an ophthalmologist and daily monitoring of the disease is required to carry out a diagnostic operation, the labour burdens and manpower shortages will inevitably surpass the existing capacity for screening. Digital imagery of the fundus and automatic or semi-automated picture analysis algorithms focused on picture processing and computer vision technologies give great potential for responding to these challenges [10, 11]. More patients can be screened and reviewed by automating the analysis process and ophthalmologists have more time to take precautions because the majority of fundus photos do not give rise to medical behavior.

\section{RELATED WORKS}

Lakshmi V [12] suggested a combination calculation based on a diagram b-shading method used in the Indian diabetic dataset Pima community. Their methodologies and KNN classification have been revised, conducted and contrasted. The results show that the combination relies on the execution of the charts as 
regards imperceptibility and accuracy rather than the other grouping approach

Rawat K., Burse K. [13] proposed Pima Indian Diabetes Dataset for Linear Discriminant Analysis and Support Vector Machine. LDA reductions involve subsets in this technique and SVM is used to order the details. A few techniques have been implemented to simplify diabetes determination for physicians and decreased the accuracy of the LDA-based diabetes dataset to make it more popular. They have then introduced and considered three separate systems, the Vector Machine, the Feed Forward Neural Network and the LDA with feed forward systems. With regard to the correlation of these methods, they argued that LDA-SVM proved to be considerably improved than the other two and is increasingly efficient because of its decreased imprevisibility. They also contrast SVM and feed the neural system (FFNN). With 2 highlights, the proposed SVM+LDA gives greater precise arrangement than $77.60 \%$.

Rajeeb Dey [14] suggested a method of evaluating diabetes mellitus by using the Artificial Neural Network Back Proliferation Calculation (ANN). The discovery of diabetes was finished in parallel, diabetic falls below class 1 and diabetic falls below class 0 . This structure is a scheme linked to the identification by using ANN history measurement of diabetes mellitus. For structure, ANN engineering is used. Random Blood Sugar Test Result, Post Plasma Blood Sugar Test, Fasting Blood Sugar Test Result and Sex, Age and their Occupation are criteria for diabetes evaluation. This system carries out 92.5 percent order execution.

Jaffar, Ali [15] suggested a diabetes-finding technique. The conclusion is completed with the estimation of the neural rear spread method. Contributions to this are plasma glucose attachment, blood pressure, triceps skin overlap, serum insulin, body mass index (BMI), family capacity diabetes amount of times a person has been pregnant and age. There was a perfect test of the missing attributes of the information collection. It has modified and acquired this framework.

Tymchenko Borys [16] suggested that early detection is one of the most critical obstacles for therapy achievement. Unfortunately, it is notoriously difficult to determine the exact stage of diabetic retinopathy Expert human understanding of fundus images is important. Simplifying the detection process is important and will benefit millions of people. Convolutionary neural networks (CNN) is successfully used in several countries adjacent subjects for diabetic retinopathy diagnosis themselves. But the high cost of large markings the efficiency of these approaches can be compromised by data sets and incoherence between the various doctors. Author propose an automated method for diagnosis of diabetic retinopathy, based on the advancement of deep learning. Via single human fundus photography. Furthermore author suggest a multi-stage transfer method training that uses different etiquette with related datasets. Junjun Pan [17] indicated that the leading cause of avoidable vision was diabetic retinopathy (DR). Manual identification of DR currently takes time task that depends on professional and well-trained clinicians. In this, Author suggest a new automated detection of diabetes retinopathy (DR) Deep convolutionary neural networks method (DCNNs). To We design an attention mechanism to classify region of interests (ROIs) to scoring regions referred to as map scoring regions there was a mistake (RSM). The RSM consists of deep neural networks, which are only trained by large-scale DR labels Data set. Data set. The RSM is inserted in the deep residual in particular intermediatestage networks. The proposal with RSM Model will highlight the various regions of a retina picture discriminatory ROIs for gravity of picture.

\section{DIABETIC RETINOPATHY}

In recent decades, there has been a significant rise in diabetes diagnosed, with diabetic retinopathy being one of the most extreme diseases, and the risk of a variety of eye diseases has increased. In addition, the primary cause of blindness in middleaged adults is diabetic retinopathy. Early detection of diabetic retinopathy, even for qualified clinicians, despite ongoing efforts, may result in delays in care and poor communications. The importance of automated retinopathy detection methods was recognised. We based in our research on classifying the retinal images in normal and the diabetic images of retinopathy (samples for our classification problems). Previous efforts have been made in using the removal of images and machine learning techniques; hard exudates, red lesions, microaneurysms and vascular detection are featured in the classification system while task classification involves (SVM). (KNN) Algorithm, k-nearest neighbour, and so on. However none of the manual features will cover all diabetic retinopathy symptoms in the picture, with a large proportion being usual and diagnosing the normal cases several times. There is a minimal successful clinical use of the method. [3]
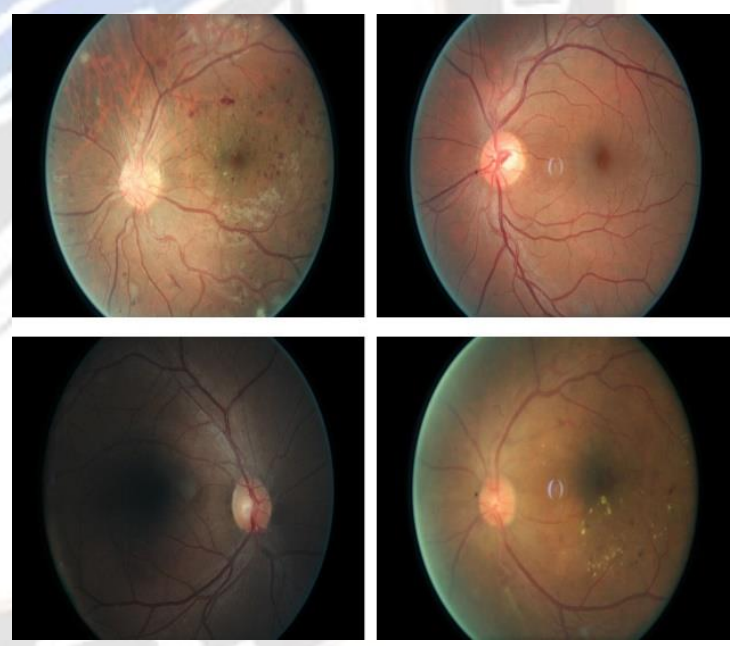

Figure 1. Sample Frames of the Retina Images

Typical subjects are the initial two edges of the top line, while the two edges of the basic column are patients with diabetic retinopathy. In many areas of PC vision, for instance, object position, picture grouping, object tracking or edge identity, the late pull propels in convolutionary neural networks $(\mathrm{CNn})$ have become the cutting-edge advancement in image characterization process. In comparison to the use of hand-made capacity that can be used for the image grouping, CNN can become familiar in a range of capabilities. Since different levelled techniques can be used in order to fit ever complex highlights, just as in higher layers representation and contortion often involve, the accuracy of CNN-based image management strategies can be improved. In view of this speculation, we explored the use of CNN-based 
techniques for preliminary diabetic retinopathy in this report. In addition, a specific multi-layer $\mathrm{CNN}$ engineering process was designed and study of genuine retina information was conducted.

The microvascular problem of diabetes that causes defects in the retina is a diabetic retinopathy. In the initial phase there are typically no critical signs, but the volume and gravity are mostly increased in time. The movement of the disease is shown in detail in the accompanying text. Diabetic retinopathy typically begins in the retinal vessels with no changes. The slightest discernible anomalous microaneurysm (MA) occurs in the retina as a small red dab that is a minimal increase in the damaged retinal vessels. These dangerous slim dividers can lead to the cracking and intraretinal discharge of small veins (HA). In the retina, a small red sprig may be the same as a microaneurysm, or it appears to be an immense, sporadic, roundabout feel. Diabetic retinopathy also improves slender retinal edoema and hard exudate penetrability (HE). Difficult exudate is an earlier lipid which slims out of the weakened veins and looks very yellow. If a vascular impediment triggers the district's narrow distribution and the oxygen back bomb, then a pale area with darkened borders appears in the retina. These lands are tiny microinfarcts known as sensitive exudates (Se). Important changes of diabatic retinopathy are shown by miniaturised vascular differences in size from the normal of the district, by slim framework dilatation and vein sores, and by changes in the blood vessel and vein form, intraretinals of smaller scale vascular abnormalities (IRMA) and virus diseases. The creation of new fragile veins contribute to an enormous number of hypoxia and hair-like blockages in the retina. These fresh recruiters try to manufacture vitamins and oxygen within a tissue-filled heading. However, fresh vessels are fragile and usually can grow into the space between retina and glassy funnality or into glassy divertisement, leading to preretinal release and sudden visual impairment. Neovascularization is the production of these fresh recruit vessels.

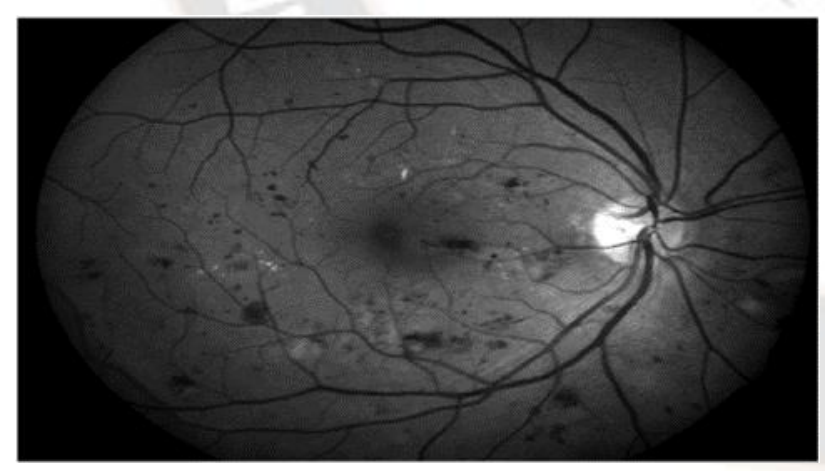

Figure 2: Examples of Pre Processed Image.

Fundus photography as mentioned above is considered as the above-mentioned method, since it is solid, unobtrusive and userfriendly to fundus photography [9]. It can record symptom details and after that, direct master meetings, as well as contrasted and common ophthalmoscopes. More critically, the speed of acknowledgement of a strange fundus could increase affinity that is the pace of acknowledgement. Duo cameras are now delivering easy to record photographs in portable environments, using image research calculations to analyse diabetic retinopathy as a result of the rapid progression of computerised imagery.

The fundus camera is divided into two meetings: extended and non mydriatic cameras, where the front picture suggests that the student must be expanded by eye drops. The pre-setting is misdirected because the production is manipulated by and for the two types of fundus camera. For screening purposes, a nonmydriatic fundus camera is compact and fair, but the photo quality is more regrettable and the field is wider. This way, an expanded camera is used when an increasingly precise decision is required. The patient sits on the headrest of the instrument before the fundus camera. The resulting light is transmitted to the eye of the patient using an optical mirror and a focal point and the camera sensor captures the reflected light.

\section{METHODOLOGIES}

The CNN is a neural system (ANN) which is essentially the same as an ordinary neural system. The neural system is the same as a neural system. CNN is a fascinating profound design in which individual neurons are carved to respond to areas in the field of view. $\mathrm{CNN}$ is a significant class of apprenticeships animated by natural neural systems. In the last couple of years, various varieties were suggested. However the basic pieces are basically the same. $\mathrm{CNN}$ provides dialogue sharing and grouping. In order to minimise calculation time and further spatial and design invariance, convolutionary layers are typically scattered with accumulation layers and are entirely connected to the one dimensional layer for the last couple of close to the yield) layers. The feedforward neural system can be seen in more detail as a capacity $f$ of the knowledge mapping $\mathrm{x}$ :

$$
f(x)=f_{L}\left(\ldots f_{2}\left(f_{1}\left(x_{1}, w_{1}\right), w_{2}\right) \ldots, w_{L}\right) .
$$

In contrast to model knowledge, the parameters can be gained with the intention of making the following capability $f$ an useful mapping. Each $\mathrm{x} 1$ is a Cluster $\mathrm{M} \times \mathrm{N}$ x C, officially. We may reduce our concern to a two-fold grouping issue. The implications are then stained so that they cover the first image in a superior way (eg, edges in the picture). The convolution layer consists of a rectangular neuron matrix which provides information on the rectangular territory of the previous layer. Each layer of convolution can also have a few cross sections using specific channels. Typically, after each convolutionary layer there is a pooling layer sub-sampled from the previous convolutionary layer. This set should be possible in a number of ways, such as midpoints, maxima etc. Finally a completely connected layer (or layers) (might be a fully linked, collected or convoluted layer) is generated with the product of the past layer to reflect the entire picture of knowledge following a few convolutionary layer and the largest set layer. Less highlights. Less highlights. Restore the system and stochastically incline plunge. Please note that forward and reverse generation depending on the form of layer.c will vary. 


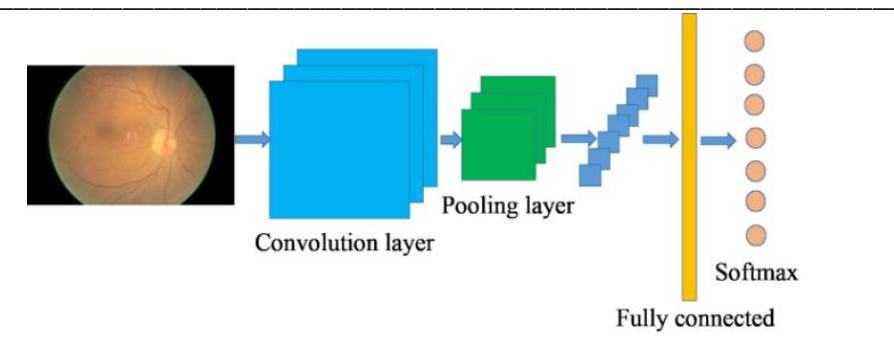

Figure.3 An Exemplary Architecture of The Convolutional Neural Network

\section{Simulation}

The data collection comprises photographs from various patient classes, the lighting of fundus photography being highly different. The lighting influences the values of the pixel intensity in the image and induces unrelated changes to the degree of classification. In order to detect and align input images with targets, a multilevel convolution neural network has been introduced. Simulation on the mathematical modelling tool MATLAB was performed and algorithms were tested for the execution time and precision of certain images.

\begin{tabular}{|l|l|}
$\underbrace{}_{\text {Data }}$ & $\begin{array}{l}\text {-Collection of Data (STARE/DRIVE) } \\
\text { Collection }\end{array}$ \\
\hline $\begin{array}{c}\text { Pre- } \\
\text { Processing }\end{array}$ & $\begin{array}{c}\text {-Pre-Processing/Blood Vessel Segmentation } \\
\bullet \text { Cotton Wool Analysis }\end{array}$ \\
\hline $\begin{array}{c}\text { Deep } \\
\text { Learning }\end{array}$ & $\begin{array}{l}\text { - Implementation of CNN } \\
\text {-Results and Classification Analysis }\end{array}$ \\
\hline
\end{tabular}

Figure.4 Process flow Diagram

VI.

\section{RESULTS}

20 topics were tested for the efficiency of the proposed network and the gradient errors were found to be good and easy to execute. In 15 epochs, an epoch of 05 seconds was completed to achieve accuracy and error threshold. Increased data size and time to achieve a fault gradient and desired accuracy would also dramatically increase the number of periods and the time taken to perform the sample. Both real-time and offline data can be used with the proposed algorithm.

Table 1

Analysis of Accuracy and Execution Time

\begin{tabular}{|l|c|c|}
\hline Database & $\begin{array}{c}\text { Percentage Accuracy } \\
\text { (Without Pre- } \\
\text { processing) }\end{array}$ & $\begin{array}{c}\text { Percentage Accuracy } \\
\text { (With Pre Processing) }\end{array}$ \\
\hline Stare & $74 \%$ & $83 \%$ \\
\hline Drive & $81 \%$ & $92.5 \%$ \\
\hline
\end{tabular}

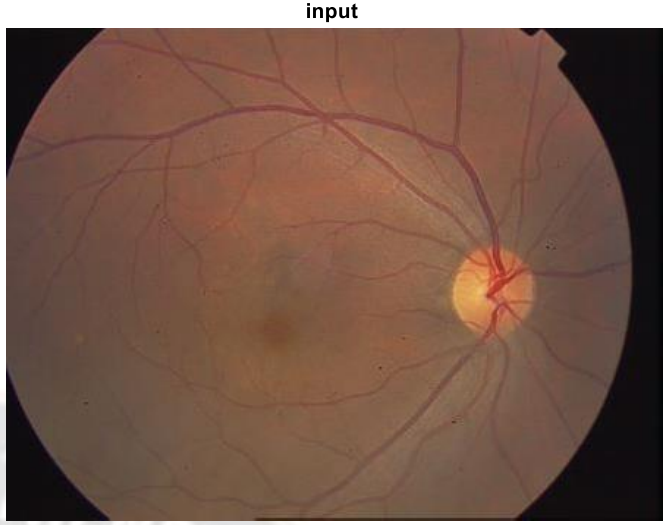

Figure.5 Sample Data Set

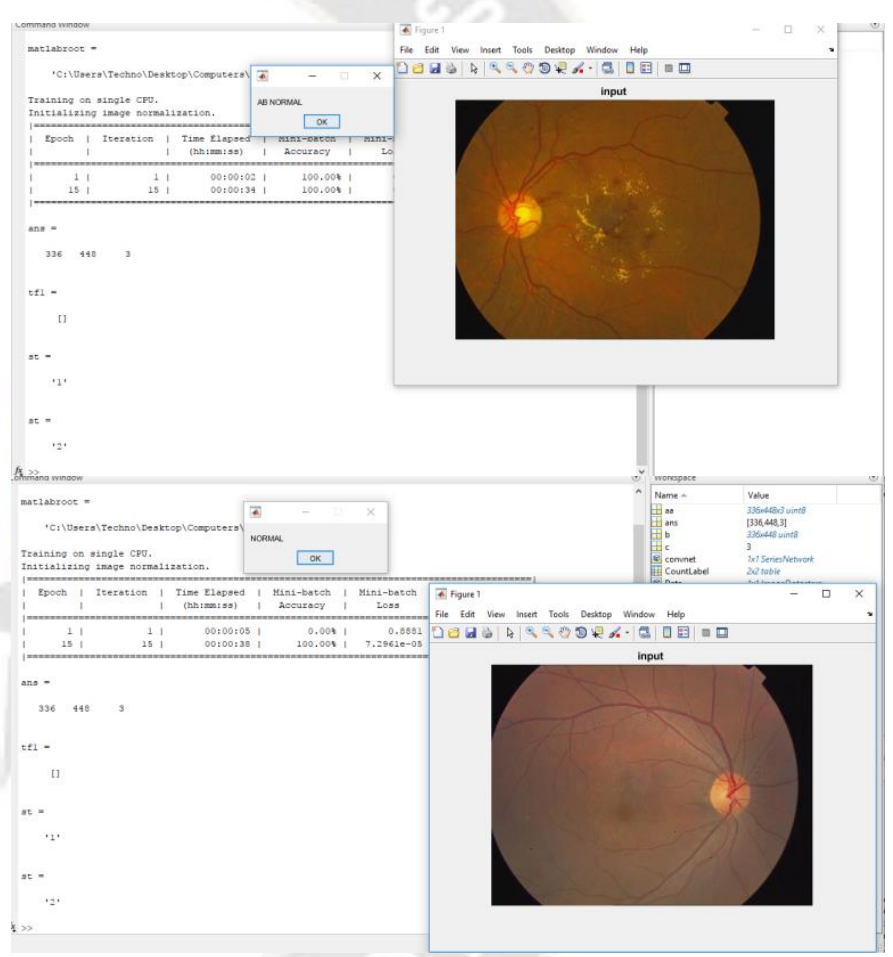

Figure.6 Detection of Normal and Abnormal Input Image Using Deep Learning CNN

Figure 5 and figure 6 show the sample dataset and execution of algorithm with respect to input data of different sets.

\section{CONCLUSION}

A rare ability to avoid most people losing view is automatic detection and screening. Scientists late added CNN to the scheme for diabetes screening estimates. CNN relies on the use of screen experts and on gaining a large amount of photographs aggregated. The original pixel. Due to the wide and low variations among these models, $\mathrm{CNN}$ may examine a wider range of non-diabetic patients the equivalent is true for diseases.

However, since we use double classifiers to carry out the most advanced presentation of CNN, the execution of the model is reduced. Increased the number of courses. Despite the fact that 
more knowledge can only be difficult to imagine, the past work is still in progress. The field shows that the ability of CNN to withstand the changes in scale is limited, others say that in this case, the qualification of mellow and typical disease is less than $1 \%$ of the total pixel amount, which is often difficult to detect by human translators.

\section{REFERENCES}

[1] M. D. Abr`amoff, J. M. Reinhardt, S. R. Russell, J. C. Folk, V. B. Mahajan, M. Niemeijer, and G. Quellec. Automated early detection of diabetic retinopathy. Ophthalmology, 117(6):1147-1154, 2010.

[2] B. Antal and A. Hajdu. An ensemble-based system for microaneurysm detection and diabetic retinopathy grading. IEEE transactions on biomedical engineering, 59(6):1720-1726, 2012.

[3] J. C. Chan, V. Malik, W. Jia, T. Kadowaki, C. S. Yajnik, K.-H. Yoon, and F. B. Hu. Diabetes in asia: epidemiology, risk factors, and pathophysiology. JAMA, 301(20):2129-2140, 2009.

[4] N. G. Congdon, D. S. Friedman, and T. Lietman. Important causes of visual impairment in the world today. Jama, 290(15):2057-2060, 2003.

[5] E. Decenci`ere, X. Zhang, G. Cazuguel, B. La“y, B. Cochener, C. Trone, P. Gain, R. Ordonez, P. Massin, A. Erginay, et al. Feedback on a publicly distributed image database: the messidor database. volume 33, pages 231-234, 2014.

[6] G. Gardner, D. Keating, T. Williamson, and A. Elliott. Automatic detection of diabetic retinopathy using an artificial neural network: a screening tool. British journal of Ophthalmology, 80(11):940-944, 1996.

[7] R. Gargeya and T. Leng. Automated identification of diabetic retinopathy using deep learning. Elsevier, 2017.

[8] J. K. H. Goh, C. Y. Cheung, S. S. Sim, P. C. Tan, G. S. W. Tan, and T. Y. Wong. Retinal imaging techniques for diabetic retinopathy screening. Journal of diabetes science and technology, 10(2):282294, 2016.

[9] B. Graham. Kaggle diabetic retinopathy detection competition report. 2015.

[10] V. Gulshan, L. Peng, M. Coram, M. C. Stumpe, D. Wu, A. Narayanaswamy, S. Venugopalan, K. Widner, T. Madams, J. Cuadros, et al. Development and validation of a deep learning algorithm for detection of diabetic retinopathy in retinal fundus photographs. JAMA, 316(22):2402-2410, 2016.

[11] L. C. Huang, C. Yu, R. Kleinman, R. Smith, R. Shields, D. Yi, C. Lam, and D. Rubin. Opening the black box: Visualization of deep neural network for detection of disease in retinal fundus photographs. The Association for Research in Vision and Ophthalmology, 2017.

[12] Lakshmi, Nadeem, Malik M. Saad Missen, and Aqsa Rashid. "Diabetic retinopathy techniques in retinal images: A review." Artificial intelligence in medicine 97 (2019): 168-188.

[13] Rawat, Kavita, and Kavita Burse. "A soft computing genetic-neuro fuzzy approach for data mining and its application to medical diagnosis." Int J Eng Adv Technol 3, no. 1 (2013): 409-411.

[14] Nath, Anirudh, Rajeeb Dey, and Carlos Aguilar-Avelar. "Observer based nonlinear control design for glucose regulation in type 1 diabetic patients: An LMI approach." Biomedical Signal Processing and Control 47 (2019): 7-15.

[15] Raza, Jaffar Ali, and Assad Movahed. "Current concepts of cardiovascular diseases in diabetes mellitus." International journal of cardiology 89, no. 2-3 (2003): 123-134.

[16] Tymchenko, Borys, Philip Marchenko, and Dmitry Spodarets. "Deep Learning Approach to Diabetic Retinopathy Detection." arXiv preprint arXiv:2003.02261 (2020).

[17] Junjun, Pan, Yong Zhifan, Sui Dong, and Qin Hong. "Diabetic Retinopathy Detection Based on Deep Convolutional Neural Networks for Localization of Discriminative Regions." In 2018
International Conference on Virtual Reality and Visualization (ICVRV), pp. 46-52. IEEE, 2018.

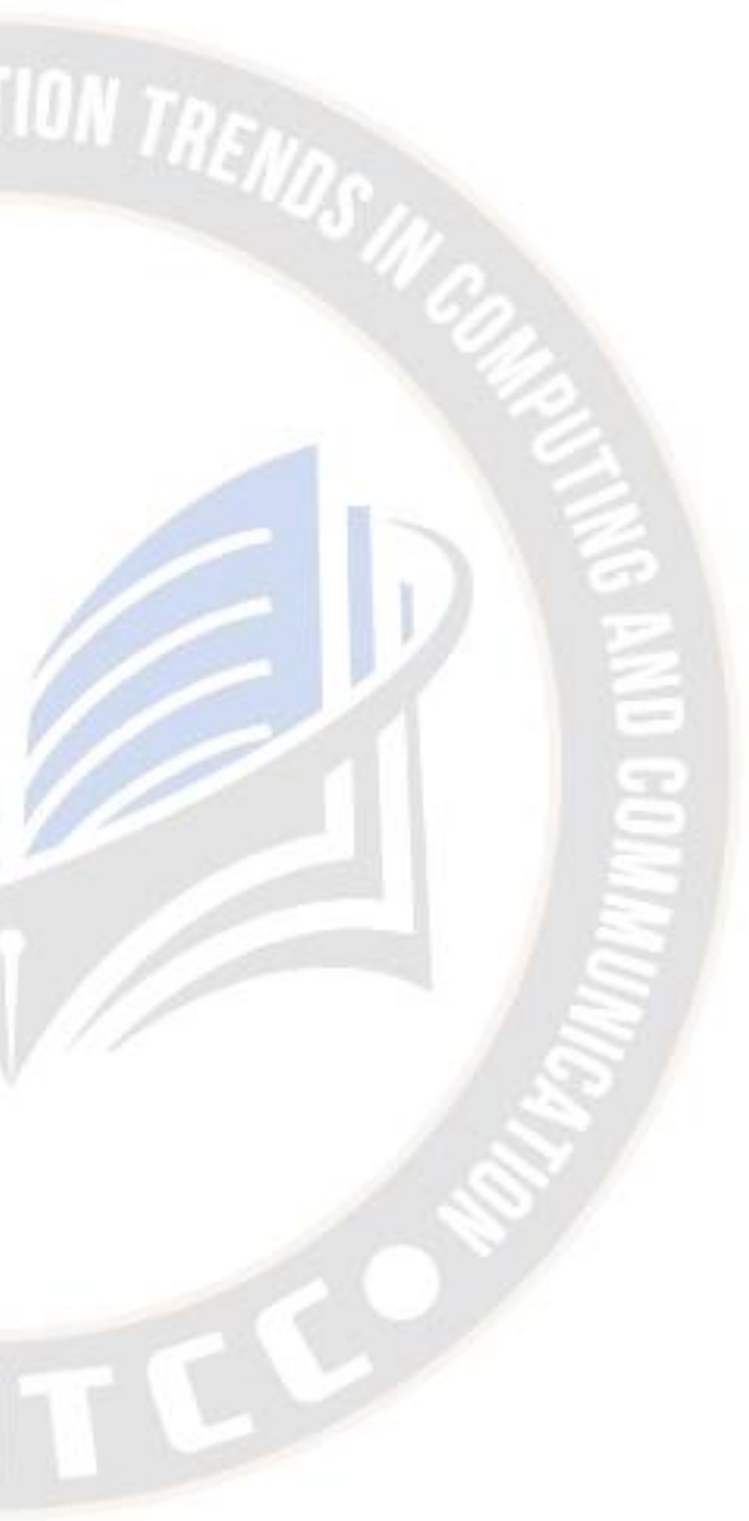

